\title{
Hemmung der Crown-Gall-Entstehung durch Fluordesoxyuridin
}

\author{
Von Martin Bopp \\ Aus dem Institut für Botanik der Technischen Hochschule Hannover \\ (Z. Naturforschg. 19 b, 64-71 [1964]; eingegangen am 10. Juli 1963)
}

\begin{abstract}
The induction of crown-galls is inhibited by 5-fluorodesoxyuridine (FUDR). This inhibition can easily be distinguished from the much smaller effect of FUDR on Tumor growth and on general plant development. The inhibition can be reversed completely only by thymidine in higher concentrations. The reversibility is restricted to the first $3-4$ days after infection of the plant. The virulence of the bacteria and the wound reaction of the plants are scarcely affected by the corresponding concentrations of FUDR. Thus the primary step in the transformation of a plant cell to a tumor cell can be selectively blocked and presupposes an undisturbed DNA synthesis, i. e. a change or activation in DNA is a part of the primary step in the transformation.
\end{abstract}

In mehreren früheren Veröffentlichungen haben wir gezeigt ${ }^{1-3}$, daß sich die Entstehung der Wurzelhalsgallen durch verschiedene Substanzen blockieren läßt, die in den DNS-Stoffwechsel der Pflanzen eingreifen. Daraus lassen sich Schlüsse auf die primäre Veränderung ${ }^{4}$ bei der Induktion von Tumoren (crown-gall) durch Agrobacterium tumefaciens ziehen. Die eindeutigste Wirkung wurde dabei mit 5-Fluordesoxyuridin (FUDR) erzielt, während z. B. die Hemmung der Gallenentstehung durch Bromuracil nur unter der Einhaltung bestimmter experimenteller Voraussetzungen gewährleistet ist ${ }^{5,6}$. Wir haben es deshalb für notwendig erachtet, die mit FUDR zu erzielende Hemmung einer genaueren Untersuchung zu unterziehen, 1. um die Selektivität auf die Gallenentstehung sicherzustellen, 2. weil die Untersuchungen an tierischen Objekten und Mikroorganismen eine unterschiedliche Reaktionsweise von FUDR und Bromdesoxyuridin (BUDR) ergeben haben. Während BUDR direkt in die DNS eingebaut wird und dadurch deren Wirksamkeit unterbindet $^{7}$, hemmt FUDR kompetitiv die Thymidylsäure-Synthetase und damit die für die DNS notwendige Thymidylsäure ${ }^{8}$. Auch für autotrophe grüne Pflanzen liegt eine solche unterschiedliche Wirkung nahe ${ }^{9,10}$, ist aber bisher nicht eindeutig bewiesen. 3. Um möglichst eine sichere Entscheidung über den Wirkungsmechanismus des FUDR bei der Gallenentstehung zu erhalten.

1 M. Bopp, Planta 54, 221 [1960].

2 M. Bopp, Z. Naturforschg. 16 b, 336 [1961].

3 M. Bopp, Jahrb. der TH, Hannover 1960/62, 208.

4 A. C. Braun, Ann. Rev. Plant Phys. 13, 533 [1962].

5 I. Lipetz u. T. Stonier, Nature [London] 190, 929 [1961].

6 M. Bopp, Z. Naturforschg. 17 b, 282 [1962].

7 u. a. K. Burton, Biochim. biophysica Acta [Amsterdam] 55, 413 [1962]. - M. T. HAKALA, Biochim. biophysica Acta [Amsterdam] 61, 815 [1962].
Die folgenden Untersuchungen sollen darum diesen Punkten gewidmet sein. Es wird darin bewiesen, daß die Hemmung der Wurzelhalsgallen durch FUDR auf einer Blockierung der DNS-Synthese beruht und diese selektiv die Entstehung der Gallen betrifft.

\section{Material und Methode}

Die Versuche wurden wie in den früheren Experimenten ${ }^{2}$ an den Pflanzen eines Klons von Kalanchoë daigremontiana R. Hamt et H. PerR de Labath im Gewächshaus durchgeführt. Zur Infektion verwendeten wir den hochvirulenten Stamm $\mathrm{B}_{6}$ von Agrobacterium tumefaciens (Conn.) Sмith and Towsend (von A. Braun, New York)*. Die Pflanzen wurden bei Versuchsbeginn an 4 Stellen auf dem obersten vollausgebildeten Blattpaar durch 2 kreuzweise Schnitte von $1 \mathrm{~cm}$ Länge verwundet und 24 Stdn. später auf dieser Verwundung mit Bakterien aus 48 Stdn. alten Agarkulturen infiziert. Dies führte zu einem durchschnittlich 100 proz. Infektionserfolg. Die Applikation des FUDR und der übrigen Substanzen erfolgte stets durch Einspritzen in das Mesophyll. In jedem Fall ist auf gleichmäßiges und gut entwickeltes Pflanzenmaterial zu achten, da schwache Pflanzen nicht nur in der Gallenbildung variieren, sondern auch sehr viel leichter durch FUDR geschädigt werden als gut entwickelte ${ }^{2}$. Bei der Versuchsanstellung ergaben sich gewisse Schwierigkeiten dadurch, daß alle injizierten Nucleinsäurebausteine sich in den Blättern nur sehr begrenzt ausbreiten und direkt zum Vegetationskegel abwandern, was bei der Auswertung der einzelnen Versuche berücksichtigt wer-

8 C. Heidelberger, L. Bosch, N. K. Chauduri u. P. B. DanNenBerg, Ferderat. Proc. 16, 194 [1957].

9 B. A. Kinlmann, Exp. Cell Res. 27, 604 [1962].

10 J. A. D. Zeevaart, Plant Phys. 37, 296 [1962].

* Herrn Dr. U. När danke ich sehr für die Übermittlung der Bakterien. 
den mußte. Zur Auswertung der Versuche wurde die Anzahl der angehenden Infektionsstellen, die allerdings bei dem hochvirulenten $B_{6}$ meist nicht wesentlich verändert war, und zum Schluß das Trockengewicht der ausgeschnittenen Tumoren bestimmt. Dieses ist abhängig von der Stärke der Umstimmung der Wirtszellen am Wundrand. Dabei sind immer nur die gleichzeitig angestellten Versuche unmittelbar miteinander vergleichbar.

Außer dem FUDR ** stammten die verwendeten Versuchssubstanzen von Nutritional Biochem. Corp., Cleveland 28, Ohio.

\section{Experimente und Ergebnisse}

Nachdem bereits in den früheren Untersuchungen gezeigt war, daß die Wirkung des FUDR konzentrationsabhängig ist ${ }^{2}$, verwendeten wir in unseren jetzigen Versuchen eine Konzentration, die eine möglichst starke Gallenhemmung, aber nur eine geringe Schädigung der gesamten Pflanzen zur Folge hat. Bei kräftig entwickelten Pflanzen konnte die Gesamtschädigung der Pflanzen praktisch vernachlässigt werden.

\section{Der Ort der FUDR-Wirkung}

In allen Experimenten wirkt das FUDR stets nur in unmittelbarer Nachbarschaft der Einspritzung und in den sich teilenden Zonen des Vegetationskegels und der jüngsten Blätter. So waren z. B. bei der Injektion von FUDR auf einer Blatthälfte nur die beiden Infektionsstellen auf dieser Seite stark gehemmt, diejenigen auf der anderen Blatthälfte dagegen kaum (Tab. 1). Dasselbe Resultat zeigt sich

\begin{tabular}{|l|c|c|c|c|c|c|}
\hline & $\begin{array}{c}\text { Anzahl der } \\
\text { Blätter }\end{array}$ & $\begin{array}{c}\text { Anzahl der } \\
\text { Gallen }\end{array}$ & $\begin{array}{c}\text { nicht ange- } \\
\text { gangene In- } \\
\text { fektions- } \\
\text { stellen }\end{array}$ & $\begin{array}{c}\text { Trocken- } \\
\text { gewicht } \\
\text { insgesamt } \\
{[\mathrm{g}]}\end{array}$ & $\begin{array}{c}\text { Trocken- } \\
\text { gewicht } \\
\text { Galle } \\
{[\mathrm{mg}]}\end{array}$ & $\begin{array}{c}\text { Trocken- } \\
\text { gewicht } \\
\text { in \% von B }\end{array}$ \\
\hline A. $\quad \begin{array}{l}\text { mit FUDR gespritzte } \\
\text { Blatthälfte } \\
\text { nicht gespritzte } \\
\text { Blatthälfte }\end{array}$ & 60 & 61 & 59 & 0,275 & 4,5 \\
\hline B. & 60 & 110 & 10 & 1,423 & 13,0 \\
\hline
\end{tabular}

Tab. 1. Lokalisation der FUDR-Wirkung auf der Seite, auf der die Substanz eingespritzt wird. Die nicht gespritzte Seite ist kaum beeinflußt. (Vgl. dazu auch Abb. 1.) - Auswertung nach 5 Wochen.

auch bei Injektion an der Blattspitze; die dort liegenden Infektionsstellen werden stärker gehemmt als in der hinteren Blatthälfte. In derselben lokal begrenzten Weise wirken auch die natürlichen Bausteine der DNS, z. B. Thymin, Thymidin und andere. Dies bedeutet, daß die Substanzen stets nur in den Zellen einen Effekt hervorrufen, in die sie direkt bzw. auf ihrem Weg zum Vegetationskegel gelangen. So erklärt es sich, daß Gallen an der Sproßachse durch Injektion von FUDR in die Blätter gehemmt werden (vgl. Abs. 4 A), Tumoren auf Blättern, die nicht gespritzt werden, sich dagegen völlig ungehindert entwickeln können ${ }^{2}$. Diese Beobachtung hat somit 2 Konsequenzen:

a) Bei der Versuchsauswertung ist stets die räumlich begrenzte Wirkung der Substanzen zu berücksichtigen (vgl. Abb. 1).

b) Die Vorgänge, die durch FUDR zu beeinflussen sind, laufen in den untersuchten Zellen selbst ab und beruhen nicht auf einer physiologischen Zustandsänderung der Gesamtpflanze.

** Das Fluordesoxyuridin (FUDR) stellte in dankenswerter Weise die Fa. Hoffmann-LaRoche, Grenzach/Baden, zur Verfügung.

\section{Die Wirkungsweise des FUDR}

Zunächst soll gezeigt werden, daß bei unseren Versuchspflanzen durch die Behandlung mit FUDR tatsächlich die DNS-Synthese und nicht andere Stoffwechselvorgänge betroffen werden. $\mathrm{Zu}$ diesem Zweck verfuhren wir nach folgendem Schema, um die durch FUDR verursachte Hemmung aufzuheben:

\begin{tabular}{|c|c|c|}
\hline $\begin{array}{l}\text { Verwundung } \\
\text { der Pflanze }\end{array}$ & 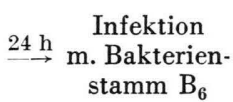 & 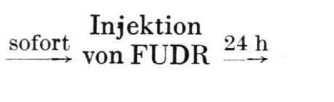 \\
\hline & & $\begin{array}{l}\text { Injektion natürl. } \\
\text { Nucleinsäurebau } \\
\text { steine und Vor- } \\
\text { stufen }\end{array}$ \\
\hline
\end{tabular}

In diesen Versuchen wurde FUDR in einer Konzentration von $10^{-4}-m$., und zwar $0,05 \mathrm{~cm}^{3}$ je Blatt $=0,1 \mathrm{~cm}^{3}$ pro Pflanze injiziert. (Dies entspricht ungefähr einer Menge von 2,4 $\gamma$ pro Pflanze.) Die Dosis der natürlichen NS-Bausteine war $5 \cdot 10^{-4}-m$., also 5-mal so groß. Die Injektion erfolgte entweder für jede Substanz getrennt auf einer Blatthälfte (z. 
B. linke Blatthälfte FUDR, rechte Blatthälfte Thymidin) oder unmittelbar nebeneinander. Die Ergebnisse waren in beiden Fällen direkt vergleichbar, wenn man das in Abschnitt 1 Gesagte berücksichtigt.

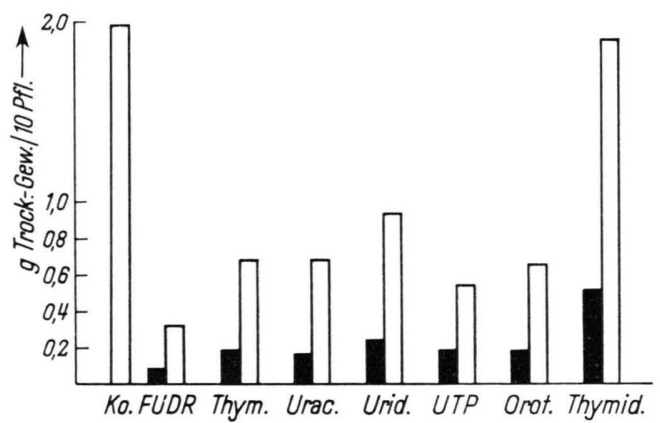

Abb. 1. Aufhebung der durch FUDR verursachten Hemmung durch verschiedene natürliche Bausteine und Vorstufen von Nucleinsäuren. Schwarze Säule die Tumorbildung auf der mit FUDR gespritzten, weiße Säule auf der mit den Antagonisten gespritzten Blattseite. $(\mathrm{Ko}=$ Kontrolle, Thym $=$ Thymin, Urac $=$ Uracil, Urid $=$ Uridin, $\mathrm{UTP}=$ Uridintriphosphat, Orot $=$ Orotsäure, Thymid=Thymidin.) Auf der Ordinate ist das Gesamttrockengewicht der an 10 Pflanzen gebildeten Tumoren aufgetragen (weitere Einzelheiten im Text).

In Abb. 1 ist das Resultat eines solchen Versuches dargestellt. Jede Säule beruht auf dem Trockengewicht der Tumoren von 40 Infektionsstellen; die schwarzen Säulen sind die jeweils auf der mit FUDR behandelten, die weißen, die auf der mit dem Antagonisten behandelten Seite gewachsenen Gallen. Bei den nur mit FUDR in eine Blatthälfte gespritzten Pflanzen beträgt das Gallenwachstum gegenüber der Kontrolle auf der behandelten Seite ungefähr 4\%; auf der anderen 16,5 Prozent. Diese sehr starke Hemmung läßt sich auf der mit Antagonisten behandelten Seite durch Thymidin vollständig aufheben, während auf der FUDR-Seite die Gallenproduktion auf etwa $24 \%$, also ebenfalls wesentlich gesteigert

\begin{tabular}{|c|c|c|c|}
\hline Vorbehandlung & $\begin{array}{l}\text { Nach- } \\
\text { behandlung } \\
{\left[5 \cdot 10^{-4}-m .\right]}\end{array}$ & $\begin{array}{l}\text { Anzahl der } \\
\text { Gallen }\end{array}$ & $\begin{array}{c}\chi^{2} \text { gegen } \\
\text { FUDR }\end{array}$ \\
\hline $10^{-4}-m$. FUDR & $\begin{array}{l}\text { Keine } \\
\text { Thymidin } \\
\text { Thymin } \\
\text { Uridin } \\
\text { Orotsäure } \\
\text { UTP } \\
\text { Uracil }\end{array}$ & $\begin{array}{l}10 \\
34 \\
22 \\
21 \\
21 \\
15 \\
11\end{array}$ & $\begin{array}{l}-\bar{c} \\
29,09 \\
7,5 \\
6,37 \\
6,37 \\
1,45 \\
0,06\end{array}$ \\
\hline
\end{tabular}

Tab. 2. Die Wirkung einer Nachbehandlung mit verschiedenen normalen Nucleinsäurebausteinen. Nur die Wirkung von Thymidin ist gut gesichert $\left(\chi^{2}>6,5\right)$. Die von Thymin war in anderen Versuchen ebenfalls nicht gesichert. Es wurden pro Blatt $0,05 \mathrm{~cm}^{3}$ der jeweiligen Lösung eingespritzt (vgl. Abb. 1). werden kann. Alle übrigen Substanzen vermögen die Hemmung kaum zu verändern. In Tab. 2 ist die Anzahl der Tumoren aus einem anderen Versuch angegeben, die Ergebnisse sind dieselben. Da also nur der unmittelbare DNS-Baustein Thymidin die FUDR-Hemmung reversibilisieren kann, und zwar quantitativ, blockiert dieses - speziell bei der Entstehung der Wurzelhalsgallen - ausschließlich die DNS-Synthese, und zwar in den zu Wurzelhalsgallen werdenden Zellen, wie wir vorher gezeigt haben.

Bereits aus der Abb. 1 kann geschlossen werden, daß zwischen FUDR und Thymidin quantitative Beziehungen zu erwarten sind. Wir kombinierten daher verschiedene Konzentrationen von Thymidin mit einer gegebenen FUDR-Konzentration.

Bei gleicher FUDR-Menge war die Größe der entstehenden Tumoren der angewandten Thymidinmenge proportional. Es ergab sich im Bereich zwischen $10^{-3}$ und $10^{-5}-m$. eine lineare Abhängigkeit zwischen dem Logarithmus der Thymidinkonzentration, die nach FUDR-Vorbehandlung gespritzt wurde und dem Trockengewicht der herangewachsenen Tumoren. Die FUDR-Konzentration war dabei

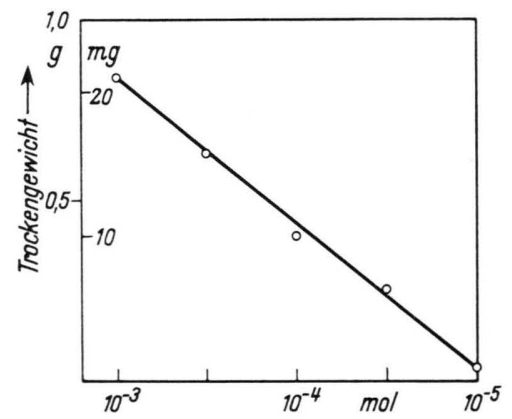

Abb. 2. Abhängigkeit der Tumorgröße an mit FUDR-gehemmten Blättern von der Thymidinkonzentration. FUDR-Menge $10^{-4}$-molar. Abszisse: Thymidinkonzentration. Ordinate: Gesamttrockengewicht (g) und Trockengewicht pro Tumor (mg). Beide Kurven sind identisch.

$10^{-4}-m$. (Abb. 2). Wenn die Thymidinkonzentration 10-mal höher liegt als die des FUDR, so wird etwa der Wert einer unbehandelten Kontrolle erreicht (vgl. Abb. 1). Gleiche Konzentration von FUDR und Thymidin führt zu Tumoren, die etwa um $50 \%$ gehemmt sind. Eine 10-mal niedrigere Thymidinkonzentration hingegen hat nahezu keinen Effekt mehr auf die FUDR-Hemmung.

Auch aus diesen quantitativen Versuchen ist der Eingriff des FUDR in die DNS-Synthese zu ersehen. Auf die Art der Wirkung wird in der Diskussion 
einzugehen sein. Für die weiteren Versuche genügt es zu wissen, daß ausschließlich die DNS von unseren Eingriffen betroffen wird.

\section{Zeitliche Abhängigkeit der FUDR-Wirkung}

Zeitversuche haben wir bereits in geringerem Umfang sowohl mit Bromuracil als auch mit FUDR durchgeführt ${ }^{1,3}$. Gerade derartige Zeitversuche können uns eine Aussage über die selektive Blockierung der Gallenentstehung liefern, weil das genaue Studium der Induktionsvorgänge gezeigt hat, daß in zeitlicher Reihenfolge qualitativ verschiedene Schritte bei der Induktion von Tumoren aufeinanderfolgen 4, 11,12. Die Versuche wurden folgendermaßen angestellt: Unmittelbar nach der Infektion injizier-

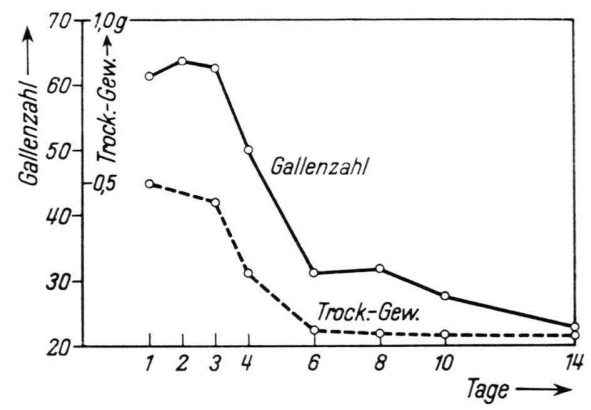

Abb. 3. Abhängigkeit der Tumorbildung an FUDR-gehemmten Blättern vom Zeitpunkt der Thymidinnachbehandlung. Abszisse: Tag der Thymidinnachbehandlung, Ordinate: Anzahl der angegangenen Infektionsstellen (von 64) bzw. Gesamttrockengewicht. FUDR $=0,4 \mathrm{~cm}^{3} 2 \cdot 10^{-5}-\mathrm{m}$. pro Pflanze, Thymidin $0,4 \mathrm{~cm}^{3} 5 \cdot 10^{-4}-\mathrm{m}$. pro Pflanze.

ten wir in dem in Abb. 3 angegebenen Versuch insgesamt je $0,4 \mathrm{~cm}^{3}$ einer $2 \cdot 10^{-5}-m$. Lösung von FUDR in die Pflanze. In anderen Versuchen haben wir die Konzentrationen etwas variiert, ohne grundsätzlich andere Ergebnisse zu erzielen. Am gleichen (1.), nächsten (2.), übernächsten (3.) usw. Tag spritzten wir dann ebenfalls $0,4 \mathrm{~cm}^{3}$ einer $5 \cdot 10^{-4}$ - $\mathrm{m}$. Thymidinlösung in dieselben Blätter ein. Das Resultat dieses Versuches zeigt Abb. 3. Die beiden Kurven für die Anzahl der Gallen und das Trockengewicht verlaufen praktisch parallel. Bis zum 3. Tag (= $48 \mathrm{Stdn}$. nach Infektion) ist die Wirkung des Thymidins konstant und die FUDR-Hemmung durch die gegebene Konzentration fast vollkommen aufgehoben. Am 4. Tag ist die Gallenzahl etwas, das Trockengewicht stärker herabgesetzt. Folgt die Behandlung am 6. Tag und später auf die vorher-

11 A. C. Braun, Proc. nat. Acad. Sci. USA 44, 344 [1958]. gegangene FUDR-Hemmung, so hat sie keinen Einfluß mehr. Die Tumoren bleiben wie bei einer reinen FUDR-Kontrolle gehemmt.

Entsprechend diesen Versuchen ist zu erwarten, daß Fluordesoxyuridin ebenfalls nur einen Hemmeffekt hat, wenn es innerhalb der ersten 3 bis 4 Tage nach der Infektion in die Blätter eingespritzt wird.

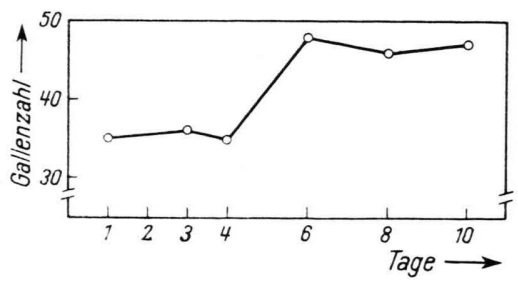

Abb. 4. Abhängigkeit der Tumorbildung vom Zeitpunkt der FUDR-Behandlung. Ordinate: Tag der FUDR-Behandlung. Abszisse: Anzahl der angegangenen Infektionsstellen (von 64). FUDR $0,4 \mathrm{~cm}^{3} 2 \cdot 10^{-5}-m$. pro Pflanze.

Das wird durch Abb. 4 bestätigt: Die Hemmung ist innerhalb der ersten 4 Tage stark, vom 6. Tag an jedoch viel schwächer. Allerdings sind auch die später mit FUDR (6 bis 10 Tage nach der Infektion) gespritzten Wurzelhalsgallen gegenüber der Kontrolle immer noch gehemmt. Dies ist jedoch nicht weiter verwunderlich, da FUDR von sich teilenden Geweben (den jungen induzierten Gallen) attrahiert wird und in diesen durch die verminderte DNS-Synthese eine gewisse Wachstumshemmung verursachen, die aber klar von der starken Blockierung der Gallenentstehung in den ersten 3 Tagen nach der Infektion zu unterscheiden ist. Die Zahlen in der Tab. 3 machen dies deutlich.

\begin{tabular}{|l|c|c|c|}
\hline $\begin{array}{c}\text { Mit FUDR } \\
\text { behandelt am }\end{array}$ & $\begin{array}{c}\text { Tumor- } \\
\text { produktion } \\
\text { pro Blatt } \\
\text { (Tumorgröße } \\
\text {-Anzahl) }\end{array}$ & $\begin{array}{c}\text { Trocken- } \\
\text { gewicht/ } \\
\text { Galle } \\
{[\mathrm{mg}]}\end{array}$ & $\begin{array}{c}\text { Anzahl } \\
\text { der In- } \\
\text { fektions- } \\
\text { stellen }\end{array}$ \\
\hline $\begin{array}{l}\text { 1. bis 3. Tag } \\
\text { nach Infektion }\end{array}$ & $2,67 \pm 0,2$ & $0,121(12 \%)$ & 120 \\
$\begin{array}{l}\text { 4. Tag nach } \\
\begin{array}{l}\text { Infektion } \\
\text { 6. bis 10. Tag } \\
\text { nach Infektion } \\
\text { Kontrolle }\end{array}\end{array}$ & $\begin{array}{c}4,32 \pm 0,57 \\
4,17 \pm 0,29\end{array}$ & $0,162(56 \%)$ & 40 \\
\hline
\end{tabular}

Tab. 3. Zeitliche Abhängigkeit der Tumorproduktion (als Maßzahl für den Erfolg der Transformation) von der FUDRBehandlung 4 Wochen nach Versuchsbeginn. Alle Pflanzen wurden nur einmal entweder am 1., 2. usw. Tag behandelt. Die gleich reagierenden Tage sind zusammengefaßt (vgl. Abb. 4).

12 R. M. Klein, Proc. nat. Acad. Sci. USA 43, 956 [1957]; 44, 350 [1958]. 
Das spätere Gallenwachstum wird von FUDR ebenfalls nur schwach beeinflußt, wie folgender Versuch zu zeigen vermag: Pflanzen wurden mit hochvirulenten Bakterien infiziert und die Gallen 4 Wochen heranwachsen gelassen. Danach wurden die Pflanzen so sortiert, daß in allen Gruppen immer dieselben Gallengrößen vorhanden waren, wodurch ein Vergleich zwischen den einzelnen Gruppen möglich war. Danach spritzten wir verschiedene Konzentrationen von FUDR in die Gallen tragenden Blätter. Bei den sonst stets verwendeten Konzentrationen von $5 \cdot 10^{-5}-m$. und $10^{-4}-m$. trat gegenüber der Kontrolle überhaupt keine Hemmung auf. Erst eine Konzentration von $5 \cdot 10^{-4}-m$. vermochte das Gallenwachstum auf etwa $75 \%$ herabzusetzen. Verglichen mit der Größe zu Versuchsbeginn sind aber auch die mit der höchsten Konzentration behandelten Wurzelhalsgallen noch um das 3-fache gewachsen, woraus also eindeutig hervorgeht, daß FUDR die wachsenden Gallen in einer Konzentration, in der es ihre Entstehung fast vollständig unterdrückt, kaum beeinflußt.

\begin{tabular}{|l|c|c|c|}
\hline & $\begin{array}{c}\text { Trocken- } \\
\text { gewicht/ } \\
\text { Galle } \\
{[\mathrm{mg}]}\end{array}$ & $\begin{array}{c}\text { Zuwachs vom Aus- } \\
\text { gangswert der } \\
\text { Kontrolle 1 }\end{array}$ \\
\begin{tabular}{l|l|} 
Trocken- \\
gewicht/ \\
Galle \\
{$[\mathrm{g}]$}
\end{tabular} & $\begin{array}{c}\text { Trocken- } \\
\text { gewicht/ } \\
\text { Galle } \\
{[\%]}\end{array}$ \\
\hline $\begin{array}{l}\text { Kontrolle 1 } \\
\text { (Versuchsbeg. }\end{array}$ & 7,7 & - & - \\
$\begin{array}{l}\text { Kontrolle 2 } \\
\text { nach 5 Wochen }\end{array}$ & 30,4 & 22,7 & 100 \\
$\begin{array}{l}\text { FUDR } \\
5 \cdot 10^{-4} m . \\
\text { nach 5 Wochen }\end{array}$ & 24,4 & 16,7 & 74 \\
$\begin{array}{l}\text { FUDR 10-4 } \\
\text { nach 5 Wochen }\end{array}$ & 28,6 & 20,9 & 92,5 \\
$\begin{array}{l}\text { FUDR } \\
5 \cdot 10^{-5} \text { m. } \\
\text { nach 5 Wochen }\end{array}$ & 34,2 & 26,5 & 116,8 \\
\hline
\end{tabular}

Tab. 4. Wirkung des FUDR auf wachsende Gallen, injiziert wurde 26 Tage nach der Infektion, und zwar $0,15 \mathrm{~cm}^{3} /$ Blatt $=0,30 \mathrm{~cm}^{3}$ pro Pflanze.

Die drei in diesem Abschnitt zusammengefaßten Versuche zeigen den engen zeitlichen Zusammenhang, der zwischen der Wirkung des FUDR bzw. der antagonistischen Wirkung des Thymidins und dem auf 3 Tage beschränkten Transformationsvorgang besteht.

13 M. Bopp, Ber. dtsch. bot. Ges. 75, 64 [1958].
Die FUDR-Hemmung der Tumoren ist nur bis zum 3. bzw. 4. Tag nach der Infektion möglich; es ist also nur ein Effekt zu erzielen, wenn die DNSSynthese innerhalb dieses Zeitraumes beeinflußt wird. Umgekehrt entstehen auch nur dann Gallen, wenn die Hemmung während dieser Zeit durch Thymidin aufgehoben wird. Danach hat eine Thymidingabe keinen Effekt mehr. Ein solcher wäre aber zu erwarten, wenn es sich lediglich um eine Wachstumshemmung im Anfangsstadium der Gallen handelte. Damit hat sich die Blockierung durch FUDR als eine echte selektive Hemmung der Gallen-Enstehung erwiesen (vgl. Bopp ${ }^{2,13,14}$ ).

\section{Einfluß des FUDR auf die Wundreaktion und die Virulenz der Bakterien}

Bevor es jedoch möglich ist, von einer Selektivität der Blockierung der primären Veränderungen der Zellen selbst zu sprechen, ist es notwendig zu untersuchen, in welcher Weise die Wundreaktion der Pflanze und die Virulenz der Bakterien durch die Behandlung mit FUDR beeinflußt werden.

A. Wundreaktion der Pflanze

Da Wundteilungen an einem Wundrand innerhalb des Blattgewebes sehr schlecht quantitativ zu erfassen sind, wurde hierfür die Versuchsanstellung in folgender Weise abgewandelt: Wir spritzten FUDR in ein Blatt ein und verwundeten die Sproßachse unmittelbar oberhalb des Blattstielansatzes durch einen $1 \mathrm{~cm}$ langen Einschnitt. Die 6 Tage nach der Behandlung fixierten Stücke der Sproßachse schnitten wir dann mit dem Mikrotom. An gleichbehandelten und mit Bakterien infizierten Pflanzen wurde außerdem die spätere Tumorentwicklung beobachtet. Bereits eine Konzentration von $8 \cdot 10^{-6} \cdot m$., in einer Menge von $0,25 \mathrm{~cm}^{3}$ unterhalb der Infektionsstelle in die Pflanzen eingespritzt, genügt, um die Gallenbildung an der Sproßachse völlig zu hemmen. Zur Auswertung wurde in den Schnitten die Anzahl der neu eingezogenen Zellwände pro Ausgangszelle in Mark und Rinde getrennt ausgezählt. Die Teilungen im Mark sind zwar für die Tumorbildung ohne Bedeutung (vgl. Bopp und Leppla ${ }^{15}$ ); ihre Wundreaktion kann aber ebenfalls als Maßstab für die Wirkung des FUDR auf diesen Vorgang dienen.

Aus der Tab. 5 ist zu entnehmen, daß trotz der Behandlung mit FUDR die Zahl der Wundteilungen

14 M. Bopp, Umschau 1962, 734. 


\begin{tabular}{|l|c|c|c|}
\hline & \multicolumn{2}{|c|}{$\begin{array}{c}\text { Durchschnittliche Anzahl } \\
\text { der Zellteilungen } \\
\text { pro Ausgangszelle }\end{array}$} & $\begin{array}{c}\text { Anzahl } \\
\text { der aus- } \\
\text { gezählten } \\
\text { Zellen }\end{array}$ \\
\hline Rinde & Mark & \\
\hline FUDR $10^{-5}-m$. & $4,35 \pm 0,17$ & $4,77 \pm 0,18$ & 100 \\
verwundet & $4,-6,39 \pm 0,19$ & $6,20 \pm 0,27$ & 75 \\
\hline
\end{tabular}

Tab. 5. Zellteilungen an Wundrändern der Sproßachse von Pflanzen, die über ein darunter liegendes Blatt mit FUDR behandelt wurden.

nur z. T. herabgesetzt ist und mindestens noch $65 \%$ der Kontrolle erreicht; sie liegt damit im gleichen Größenbereich wie die reine Wachstumshemmung durch FUDR an bereits wachsenden Tumoren.

Nach 5 Wochen waren an den infizierten und mit FUDR behandelten Pflanzen normale Wundcalli entstanden, die aus Reihen von regelmäßig angeordneten Zellen bestanden und sich eindeutig von einem Tumor unterscheiden (Bopp und Leppla ${ }^{15}$ ). Sie sehen genau wie unbehandelter Wundcallus aus. Damit ist gezeigt, daß die Zellteilungen als Folge der Wundreaktion durch Konzentrationen des FUDR, die die Tumorbildung völlig unterbinden, nur unwesentlich beeinflußt werden. Das Ausbleiben der Tumoren an Blättern, die mit FUDR behandelt sind, kann also nicht eine Folge der gehemmten Wundteilung sein.

\section{B. Die Virulenz der Bakterien}

Schließlich haben wir eine größere Anzahl von Versuchen unternommen, um zu sehen, ob sich die Virulenz der Bakterien durch Fluordesoxyuridin beeinflussen läßt. Die Resultate waren stets dieselben, weshalb wir uns auf die Ergebnisse weniger Versuche beschränken können (Tab. 6 a, b). So hatte z. B. eine Konzentration von $5 \cdot 10^{-5}-m$. FUDR, in der die Bakterien 48 Stdn. gewachsen sind, zwar die

\begin{tabular}{|c|c|c|c|}
\hline & $\begin{array}{l}\text { Wachstum } \\
\text { nach } \\
48 \text { Stdn. }\end{array}$ & $\begin{array}{c}\text { Gallenzahl } \\
\text { nach } \\
\text { 24 Tagen }\end{array}$ & $\begin{array}{l}\text { Trocken- } \\
\text { gewicht } \\
\text { mg/Galle } \\
\text { nach } \\
24 \text { Tagen }\end{array}$ \\
\hline $\begin{array}{l}\text { Kontrolle } \\
\text { FUDR }\end{array}$ & $\begin{array}{r}13 \cdot 10^{8} \\
2 \cdot 10^{8}\end{array}$ & $\begin{array}{l}64 \\
64\end{array}$ & $\begin{array}{l}5,5 \\
5,3\end{array}$ \\
\hline
\end{tabular}

Tab. 6 a. Bakterienwachstum und Virulenz der Bakterien nach 48 Stdn. Kultur in einer $5 \cdot 10^{-5}-m$. FUDR-haltigen Nährlösung. Für das Wachstum ist die Anzahl der Bakterien/ccm nach 48 Stdn. angegeben.

\begin{tabular}{|c|c|c|}
\hline Anzahl der Gallen & nach 14 Tagen & nach 24 Tagen \\
\hline Kontrolle & 40 & 40 \\
eine Passage & 37 & 40 \\
zwei Passagen & 4 & 24 \\
drei Passagen & 0 & 10 \\
\hline
\end{tabular}

Tab. 6 b. Virulenz von Bakterien, die ein oder mehrere Passagen von jeweils $48 \mathrm{Stdn}$. in einer $10^{-5}-m$. FUDR-haltigen Nährlösung gewachsen sind.

Wachstumsrate wesentlich herabgesetzt; es zeigt sich aber keinerlei Tumorhemmung, wenn Kalanchö̈Pflanzen mit solchen Bakterien infiziert werden. Anzahl und Trockengewicht der Gallen ist genau so groß wie in den Kontrollen. Die Virulenz der Bakterien geht also innerhalb $48 \mathrm{Stdn}$. (= einer Passage) nicht verloren. Auch wenn die Bakterien über mehrere Passagen in FUDR-haltiger Nährlösung kultiviert wurden, machte sich in der 2. Passage nur ein schwacher Virulenzverlust bemerkbar, der erst in der 3. Passage stärker ansteigt. Hier ist jedoch die Gesamtzahl der Bakterien in der zur Infektion verwendeten Suspension durch die Wachstumshemmung schon so stark herabgesetzt, daß ein direkter Vergleich mit der Kontrolle nicht mehr möglich ist.

Für unsere Versuche genügt es aber, die Bakterien nach einer Passage zu vergleichen, da nach den früheren Experimenten die Wirkung des FUDR nach 3 Tagen abgeschlossen ist (vgl. Abb. 3 und 4). Einen weiteren Hinweis darauf, daß die Bakterien durch FUDR innerhalb kürzerer Zeit nicht beeinflußt werden, ergibt sich aus einem Vergleich mit unseren früheren Beobachtungen ${ }^{2}$ (dortige Tab. 9) : $\mathrm{Da}$ die Bakterien in den Interzellularen bleiben ${ }^{16}$ ), könnte nur FUDR wirken, das ebenfalls in den Interzellularen verbleibt. Aus unseren jetzigen Versuchen ist zu schließen, daß bei Injektion von FUDR 3 Tage vor der Infektion im Augenblick der Infektion selber in den Interzellularen kein FUDR mehr vorhanden ist. Wenn eine Hemmwirkung beobachtet werden kann, so kann diese nur auf Vorgänge innerhalb der Zelle, nicht jedoch auf die in den Interzellularen verbleibenden Bakterien zurückzuführen sein. In den genannten Versuchen war nun die FUDR-Hemmung gleich, unabhängig davon, ob die Substanz 3 Tage vor oder 1 Tag nach der Infektion in die Pflanzen eingespritzt wird. Wir können deshalb einen Einfluß des FUDR auf die Virulenz der Bakterien während der Induktionszeit ausschließen.

16 T. Stonier, Amer. J. Bot. 43, 647 [1956].

15 M. Bopp u. E. Leppla, Planta 1964, im Druck. 


\section{Diskussion}

Nach den Untersuchungen von Heidelberger und Mitarbb. ${ }^{17,18}$ wird FUDR nicht in die DNS eingebaut, so daß fraudulente DNS entsteht, sondern die Substanz blockiert mit hoher Spezifität nach der Phosphorilierung ${ }^{19}$ durch kompetitive Hemmung die Thymidylsäuresynthetase, die für die Umwandlung von Desoxyuridylsäure in Thymidylsäure verantwortlich ist, und damit die DNS-Synthese. Das ist $u$. a. bei Knochenmarks- ${ }^{20}$, Krebs- ${ }^{17}$ und HeLazellen $^{32,33}$, bei Bakterien, Seeigelembryonen ${ }^{21}$ und Mausfibroblasten ${ }^{22}$ gezeigt worden. Für autotrophe grüne Pflanzen wird zwar ebenfalls angenommen, daß FUDR nicht in DNS eingebaut wird ${ }^{10,23}$, vor allem deshalb, weil sich Bromdesoxyuridin, dessen Einbau bekannt ist, und FUDR hinsichtlich ihrer cytologischen Wirkung nach Röntgenbestrahlung unterscheiden ${ }^{9}$. Ein eindeutiger Beweis für diese Annahme ist aber bisher nicht erbracht.

Unsere Versuche über die quantitative Beziehung zwischen Thymidin und FUDR lassen es wahrscheinlich erscheinen, daß FUDR eine Fermentblockade setzt, die durch Thymidin umgangen wird, wodurch eine \pm normale DNS-Syntheserate möglich ist. Aus der Größe der entstehenden Gallen läßt sich ableiten, daß die DNS-Syntheserate direkt von der applizierten Thymidinmenge abhängt. Allerdings bereitet unter diesen Umständen die recht geringe Wirkung des Thymins in unseren Versuchen gewisse Verständnisschwierigkeiten, da dieses von den Pflanzen sicher als Vorstufe für die DNS-Synthese verwertet werden kann, was durch seine antagonistische Wirkung zum Bromuracil zum Ausdruck kommt ${ }^{2}$. In den vorliegenden Experimenten kann es aber den Syntheseblock offenbar nicht in genügender Weise umgehen, da es sich in seiner sehr geringen Wirkung nicht von denen der übrigen DNS-Bausteine und deren Vorstufen oder verwandter Substanzen unterscheidet.

Die Annahme einer DNS-Hemmung setzt voraus, daß nach den hier vorliegenden Versuchen die $\mathrm{Nu}$ cleotid-Bausteine der DNS in den Zellen, die DNS synthetisieren, selbst hergestellt und nicht transpor-

17 C. Heidelberger, N. K. Chauduri, P. Dannenberg, D. Mooren, L. Griesbach, R. Duschinsky, R. J. Schnitzer, E. Pleven u. J. Scheiner, Nature [London] 179, 663 [1957].

19 C. Heineiberger, L. Griesbach, B. J. Montag, D. Mooren, O. Cruz, R. J. Schnitzer u. E. Grünberg, Cancer Res. 18, 305 [1958].

19 K. U. Hartmann u. C. Heidelberger, J. biol. Chemistry 236, 2006 [1961]. tiert werden, weil sonst die lokal begrenzte Blockade stets durch Bausteine aus nicht blockiertem Nachbargewebe aufgehoben werden müßte, was nicht der Fall ist (vgl. dazu Diskussion bei Bopr ${ }^{2}$ ).

Eine endgültige Entscheidung darüber, ob FUDR in grünen Pflanzen, in die DNS eingebaut wird, oder über eine Ferment-Hemmung wirkt, kann wohl nur durch markierte Bausteine getroffen werden. Auf jeden Fall beweisen die Versuche, daß es sich um einen Einfluß auf die DNS handelt. Die zeitliche Übereinstimmung der FUDR-Hemmung mit unseren früheren Versuchen ist um so eindrucksvoller, wenn wir eine DNS-Synthese-Blockierung annehmen, da dann die Bildung einer fraudulenten DNS und die teilweise Blockade der DNS auf die Entstehung der Wurzelhalsgallen denselben Effekt haben. Eine weitere Wirkungsmöglichkeit der FUDR diskutieren neuerdings Studzinski und Love ${ }^{32}$.

Wir haben im experimentellen Teil gezeigt, daß es möglich ist, durch FUDR die Entstehung von Wurzelhalsgallen, die durch die in den ersten 3 bis 4. Tagen erfolgende „primäre Veränderung“ der Wirtszellen bedingt ist, selektiv zu hemmen. Dieselbe Konzentration des FUDR, die das Wachstum bzw. die Anzahl der Wundteilungen nur um etwa $30 \%$ herabsetzt, hat eine nahezu vollständige Unterdrückung der Tumorentstehung zur Folge. Aus der unterschiedlichen Reaktion der Zellteilungsvorgänge einerseits (30\% Hemmung) und der primären Veränderung andererseits (90 bis 100\% Hemmung) folgt die unterschiedliche Empfindlichkeit beider Vorgänge auf FUDR, und damit die Selektivität der Hemmung.

Die Anzahl der Teilungen am Wundrand oder das noch stattfindende Wachstum bereits entstandener Tumoren kann als Maß für die unter FUDR-Einfluß (einer entsprechenden Konzentration) noch mög. lichen DNS-Syntheserate angesehen werden. Bei $30 \%$ Teilungshemmung bedeutet das also - grob geschätzt - eine Verminderung der DNS-Synthese um ebenfalls etwa $30 \%$ in der entsprechenden Zeit. Bereits eine solche Verminderung der DNS-Syntheserate genügt aber, um eine vollständige selektive

\footnotetext{
20 J. C. Wilson u. J. S. Dinning, Proc. Soc. exp. Biol. Med. 108, 366 [1961].

21 D. A. Karnofsky u. R. S. Basch, J. biophysic. biochem. Cytol. 7, 61 [1960].

22 J. Paul u. A. Hagrwara, Biochim. biophysica Acta [Amsterdam] 61, 243 [1962].

23 J. Bonner u. J. A. D. Zeevaart, Plant Physiol. 37, 43 [1962].
} 
Blockierung der Gallenentstehung zu erreichen. Dies ist nur dann verständlich, wenn während der primären Veränderung in den Wirtszellen DNS eine Rolle spielt, die durch eine solche 30-proz. Hemmung völlig blockiert wird, was wiederum nur möglich ist, wenn sich die für die Transformation verantwortliche DNS von der übrigen unterscheidet. Ein solcher Unterschied könnte beruhen: 1. Auf einer neuentstehenden DNS, die zum Zeitpunkt der Transformation in die Wirts-DNS einbezogen oder eingebaut wird. Für Pneumococcus ist derartiges bewiesen ${ }^{24}$. 2. Auf extra-chromosomaler DNS, wie es von RoBson und Mitarbb. ${ }^{25}$ erörtert wurde, die annehmen, daß solche extra-chromosomale DNS während der "Transformation" eine Funktion hat, da sie in Crown-gall-Gewebe in vitro einen offenbar erhöhten Anteil extra-chromosomaler DNS gefunden haben. 3. Schließlich könnte auch extra-nukleare DNS eine Rolle spielen, für deren Existenz in neuester Zeit Wollgienn und Mothes ${ }^{26}$ an jungen sich teilenden Chloroplasten Beweise erbracht haben.

Als wesentlichstes Resultat betrachten wir die Ergebnisse unserer Zeitversuche, die eine Begrenzung der durch FUDR und Thymidin beeinflußbaren Prozesse auf 3 bis höchstens 4 Tage nach der Infektion ergaben. Wird nach diesem Zeitraum durch Thymidinbehandlung auch die volle DNS-Synthese wieder ermöglicht, so entstehen dennoch keine Tumoren mehr. Da eine Nachbehandlung FUDR-gehemmter Tumoren mit Thymidin bis zum 3. Tag nach der Infektion immer denselben gleich starken Effekt hat, d. h. eine Ermöglichung der DNS-Synthese nur am 3. Tag ebenfalls zu vollkommenen Tumoren führt, andererseits eine Behandlung durch FUDR erst vom 3. Tag an zur gleichen Gallenhemmung führt wie am 1. und 2. Tag, folgt daraus, daß es auf die Vorgänge etwa $72 \mathrm{Stdn}$. nach der Infektion ankommt. Dieser Zeitraum ist als die eigentliche Transformationszeit anzusehen. Er stimmt gut mit Zeiten überein, die in den letzten Jahren durch andere Versuche abgegrenzt wurden: So stellt z. B. BRAUN 28

24 S. J. LAAK, J. molecular Biol. 5, 119 [1962].

25 H. H. Robson, M. A. Budd u. H. T. Yost, Plant Physiol. 34 435 [1959].

26 R. Wollgiehn u. K. Mothes, Naturwissenschaften 50, 95 [1963].

27 S. Kupila u. H. Stern, Plant Physiol. 36, 216 [1961].

28 A. C. Braun u. T. Stonier, Protoplasmatologia 10/5 a, 55 [1958]. fest, daß die Gallen ein Maximum an Größe erreichen, wenn die Infektion bis $60 \mathrm{Stdn}$. nach der Verwundung dauert. KLEIN und LINK ${ }^{29}$ glauben aus der Interpretation zahlreicher Versuche für die Induktionsphase einen Zeitraum zwischen 24 und 72 Stdn. abgliedern zu können, und Kupila und STERN ${ }^{27}$ finden etwa $48 \mathrm{Stdn}$. nach der Verwundung, unabhängig davon, ob die Wunde infiziert ist oder nicht, ein Maximum der DNS-Synthese.

Gerade diese erhöhte DNS-Synthese scheint uns von besonderer Bedeutung zu sein, denn nach Versuchen von ZeEvaArt 9, 30 über die Blütenbildung von Pharbitis nil, ist für die Aktivierung neuer Gene (derjenigen für den Blühvorgang) DNS-Synthese notwendig. Wird die DNS-Synthese zur Zeit der Ankunft des Blühstimulus am Vegetationskegel durch FUDR unterdrückt, erscheinen keine Blüten. Entsprechend kann man auch unsere Versuche interpretieren: Nur dann, wenn die DNS-Synthese nach der Verwundung in ihrer vollen Stärke stattfinden kann, wird auch die für die Transformation verantwortliche DNS aktiv - läuft die DNS-Synthese z. T. gehemmt ab, unterbleibt die Aktivierung dieser DNS.

Die erweiterten Versuche mit FUDR führen damit zu einer Vertiefung der Vorstellung, die wir auch schon auf Grund unserer früheren Ergebnisse entwickelt haben und entsprechen den dort aufgestellten Erwartungen: Die primäre Veränderung der Wirtszelle zur Tumorzelle muß darauf beruhen, daß während der durch die Wundteilung angeregten DNS-Vermehrung eine neue DNS aktiv wird. Damit ist die zeitlich scharf umgrenzte, primäre Veränderung als eine Art Transformationsvorgang zu betrachten, und die Entstehung der Wurzelhalsgallen stellt ein Aktivwerden sonst nicht nachweisbarer Gene dar. Dies zieht alle in vitro festzustellenden Wachstums- und Stoffwechseländerungen ${ }^{31}$ nach sich.

Die Untersuchungen wurden mit Hilfe der D e u t $\mathrm{sch}$ en Forschungsgemeinschaft durchgeführt. Fräulein Elma Scharfenorth danke ich für ihre unermüdliche und sorgfältige Hilfe bei den Versuchen.

29 R. M. Klein u. G. K. K. Link, Quart. Rev. Biol. 30, 207 [1955].

30 Vgl. auch I. Brown, Plant Physiol. 37, XII, 1962 (Supplement).

31 A. C. Braun u. H. N. Wood, Proc. nat. Acad. Sci. USA 48, 1776 [1962].

32 G. P. Studzinski u. R. Love, Exp. Cell Res. 32, 190 [1963].

33 N. P. Salzmann u. E. D. Gebring, Biochim. biophysica Acta [Amsterdam] 61, 406 [1962]. 